\title{
MicroRNA-27a activity is not suppressed in porcine oocytes
} Li Chen ${ }^{1}$, Xiaoxiang $\mathrm{Hu}^{1}$, Yunping $\mathrm{Dai}^{2}$, Qinghe $\mathrm{Li}^{1}$, Xiaobo Wang ${ }^{3}$, Qiuyan $\mathrm{Li}^{2}$, Kai Xue ${ }^{4}$,Yan $\mathrm{Li}^{2}$, Jingwen $\mathrm{Liang}^{1}$,
Yuanyuan Wang ${ }^{1}$, Xiaojuan Liu ${ }^{1}$, Ning Li

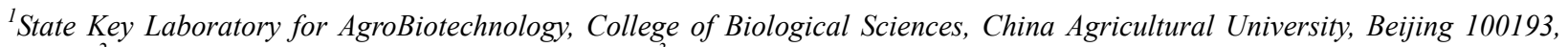
China, ${ }^{2}$ Ji Pulin biotech Ltd, Beijing 100193, China, ${ }^{3}$ Center for Cardiovascular Sciences, Albany Medical College, Albany, NY 12208, ${ }^{4}$ Dalian Medical University, Dalian 116044, China

\section{TABLE OF CONTENTS}

1. Abstract

2. Introduction

3. Materials and methods

3.1. Oocyte collection and culture

3.2. Evaluation of meiotic stages of porcine oocytes

3.3. MicroRNA expression profiling assay and analysis

3.4. Taqman real-time PCR of miRNAs

3.5. Bioinformatics method

3.6. Microinjection

3.7. Western blot analysis

3.8. Transfection and luciferase assay

3.9. Statistical analysis

4. Results

4.1. Meiotic stages of porcine oocytes during in vitro maturation

4.2. miRNA expression profiling during porcine oocyte maturation

4.3. $\mathrm{miR}-27$ a facilitates the first cell cleavage

5. Discussion

4.4. $m i R-27$ a represses translation of MAP2K4

6. Acknowledgment

7. References

\section{ABSTRACT}

MicroRNAs (miRNAs) are a class of small noncoding RNAs involved in multiple cellular processes. Recent findings indicate that miRNA activity is globally suppressed in mouse oocytes. However, whether miRNAs are expressed and function in porcine oocytes remains unknown. In this study, our aims were to ascertain if miRNA biogenesis occurs and whether miRNA activity is globally suppressed during porcine oocyte maturation. First, to identify if miRNA biogenesis occurred, a TaqMan low-density array containing 365 mature human miRNAs was used to examine miRNA expression. This analysis revealed dynamic changes in miRNA expression, suggesting miRNA biogenesis during porcine oocyte maturation. Then, to identify if miRNA activity was globally suppressed in porcine oocytes, we focused on miR-27a, which functions in the cell cycle. miR-27a was found to facilitate the first cleavage and repress the translation of its messenger RNA target (MAP2K4), suggesting that miR-27a activity was not suppressed in porcine oocytes. Taken together, our results suggest that miRNA activity was not globally suppressed in porcine oocytes, at least for miR-27a. However, because we only investigated the activity of miR-27a, further experiments are definitely required to ascertain this point.

\section{INTRODUCTION}

In mammals, oocyte maturation is crucial for species survival, but the process of oocyte maturation is complex. In most mammals, fully grown oocytes are arrested in prophase of the first meiosis, which is referred to as the germinal vesicle (GV) stage and is characterized by a large nucleus (1). In response to specific signals such as gonadotropins (2), the immature oocytes resume meiosis, defined by germinal vesicle breakdown (GVBD). Then, the first polar body is extruded, and oocytes enter and arrest the cell cycle at meiosis II (MII) until they are stimulated by a sperm (3). During these processes, oocytes are transcriptionally quiescent (4); thus, post-transcriptional regulation may be important for oocyte maturation.

MicroRNAs (miRNAs) are a class of small noncoding RNAs (approximately 22 nucleotides) that regulate gene expression at a post-transcription level by translation repression or target degradation $(5,6)$. As newly identified regulators, miRNAs are critical for oogenesis and early embryo development. For example, miRNA expression has been shown to change dynamically during mouse oogenesis and bovine oocyte maturation, suggesting potential roles of miRNAs in these processes $(7,8)$. The miRNA pathway partially inhibits accumulation of the 
rp-S2 protein during Drosophila oocyte maturation (9). However, different studies found that miRNA function was lost in mouse oocytes and early embryos. miRNAs were shown to poorly repress their targets in mouse oocytes (10). Dgcr8-deficient oocytes, which lost canonical miRNAs, matured and fertilized normally, producing seemingly healthy offspring (11). Despite having been studied in many organisms, whether miRNAs express and function during porcine oocyte maturation remains unknown. The current study was therefore designed to investigate miRNAs in porcine oocytes.

\section{MATERIALS AND METHODS}

\subsection{Oocyte collection and culture}

All the ovaries were collected from prepubertal gilts at a local slaughterhouse and transported to the laboratory within $2 \mathrm{~h}$ at $37^{\circ} \mathrm{C}$. The oocytes were aspirated from the antral follicles (3-6 $\mathrm{mm}$ in diameter) using an 18-gauge needle fixed to a $10-\mathrm{mL}$ disposable syringe. Compact cumulus-oocyte complexes (COCs) were selected, washed, and cultured for up to $44 \mathrm{~h}$ at $39^{\circ} \mathrm{C}$ and $100 \%$ humidity under $5 \% \quad \mathrm{CO}_{2}$. For the maturation culture, improved TCM-199 (Gibco, Grand Island, NY, USA) supplemented with $10 \%(v / v)$ fetal bovine serum (FBS), $10 \%(v / v)$ porcine follicular fluid, $0.57 \mathrm{mmol} / \mathrm{L}$ cysteine, $10 \mathrm{ng} / \mathrm{mL}$ epidermal growth factor, $10 \mathrm{IU} / \mathrm{mL}$ pregnant mare serum gonadotropin, and $10 \mathrm{IU} / \mathrm{mL}$ human chorionic gonadotropin was used.

\subsection{Evaluation of meiotic stages of porcine oocytes} Oocytes were evaluated for their nuclear status according to the procedures described by Sun et al. (12).

\section{3. miRNA expression profiling assay and analysis} Seventy oocytes were lysed at $95^{\circ} \mathrm{C}$ for $5 \mathrm{~min}$ and reverse transcribed using a Multiplex RT set pool (Applied Biosystems, CA, USA). Complemetary DNA (cDNA) was then loaded onto a TaqMan low-density array (TLDA; Applied Biosystems) containing 365 miRNAs in addition to 3 small nucleolar RNAs that function as endogenous controls (RNU44, RNU48, and RNU6B) according to the manufacturer's protocol. All the miRNAs were then assayed using an ABI 7900 HT Fast Real-Time Polymerase Chain Reaction (PCR) system (Applied Biosystems). Relative miRNA expression data were analyzed using the $\Delta \Delta \mathrm{Ct}$ method. RNU6B was used as the internal control, and oocytes at $0 \mathrm{~h}$ were used as a calibrator. The normalized data from the assays were then subjected to hierarchical clustering using 2 methods: (1) mean-centering data for each miRNA (but not the samples) followed by correlation similarity metrics for miRNA and sample clustering, and (2) euclidean and correlation similarity metrics to cluster miRNAs and samples, respectively, without centering the data.

\subsection{TaqMan real-time PCR of miRNAs}

The oocytes were treated as described above. The TaqMan miRNA kit (Applied Biosystems) was used to quantify the miRNA (RNU6B, let-7g, miR-21, miR-19a, and miR-27a) expression according to the manufacturer's protocol. Relative miRNA expression data were analyzed using the $\Delta \Delta \mathrm{Ct}$ method. RNU6B was used as the internal control to determine relative miRNA expression.

\subsection{Bioinformatics method}

Target genes were predicted using the 3 most known target prediction databases: TargetScan 5.1 (http://www.targetscan.org), miRBase (http://microrna.sanger.ac.uk/targets/v5), and PicTar (http://pictar.bio.nyu.edu). Pathway analysis using the KEGG Orthology-based Annotation System software was conducted to determine the intersection of the targets obtained by the 3 algorithms.

\subsection{Microinjection}

The concentration of each type of RNA (miR-27a mimics, inhibitors, or scrambles; Ambion, Inc.) in the solutions was adjusted to $0.5 \mathrm{mg} / \mathrm{mL}$. The microinjection was performed in $150 \mu \mathrm{L}$ of the culture medium using microinjectors (IM-5B; Narishige, Tokyo, Japan) equipped with manipulators (MMN-1; Narishige) mounted on an inverted microscope (Diaphoto200; Nikon, Kawasaki, Japan). Approximately $50 \mathrm{pL}$ of the RNA solution was injected into the cytoplasm of the noncultured COCs or MII oocytes by continuous pneumatic pressure. After microinjection, the COCs were cultured for $44 \mathrm{~h}$ and denuded for Western blot analysis. The MII oocytes were parthenogenetically activated as described by Wei et al. (13). Scrambles were used as the negative control in the experiment.

\subsection{Western blot analysis}

For detection of MAP2K4, oocytes or cell lines were dissolved in lysis buffer ( $80 \mathrm{mM}$ b-glycerophosphate, 25 $\mathrm{mM} \quad p$-nitrophenylphosphate, $\quad 25 \quad \mathrm{mM}$ 3-Morpholinopropanesulfonic Acid ( $\mathrm{pH}$ 7.2), $20 \mathrm{mM}$ ethylene glycol tetraacetic acid, $20 \mathrm{mM} \mathrm{MgCl}{ }_{2} \cdot 6 \mathrm{H}_{2} \mathrm{O}, 1.1$ $\mathrm{mM} \mathrm{Na} \mathrm{VO}_{4}, 1 \mathrm{mM}$ phenylmethanesulfonyl fluoride, 2 $\mu \mathrm{g} / \mathrm{mL}$ aprotinin, $200 \mu \mathrm{M}$ leupeptin, $5 \mathrm{mM} \mathrm{NaF}$, and $1.0 \%$ NP-40). The protein samples were denatured at $98^{\circ} \mathrm{C}$ for 10 min and then separated by $10 \%$ sodium dodecyl sulfate-polyacrylamide gel electrophoresis gel and transferred to a polyvinylidene fluoride membrane. After the transfer, the membranes were blocked with Tris-buffered saline containing $0.1 \%$ Tween-20 and 5\% low-fat dry milk and probed using primary antibodies. Antibodies against MAP2K4 (Abcam, Inc., UK) and $\beta$-actin (Santa Cruz Biotechnology, Inc., USA) were used. Finally, the results were obtained using horseradish peroxidase-linked secondary antibodies (Sigma-Aldrich, USA) and enhanced chemiluminescence.

\subsection{Transfection and luciferase assay}

The 293FT cells were maintained in Dulbecco's Modified Eagle's Medium (Sigma-Aldrich, USA) supplemented with $10 \%$ FBS (Gibco, USA) and maintained at $37^{\circ} \mathrm{C}$ in the presence of $5 \% \mathrm{CO}_{2}$. Pig $M A P 2 K 4$ 3 '-untranslated region (UTR) was amplified from the cDNA of the pig ovary using primers 5'-CCCAGACACCATGTGCAATAAG-3' and 5'-GGTGCGTTTTATTACAACTGACC-3', cloned into the EZ-T Vector (GenStar BioSolutions, China), and then finally subcloned into the psiCHECK-2 Vector (Promega, 


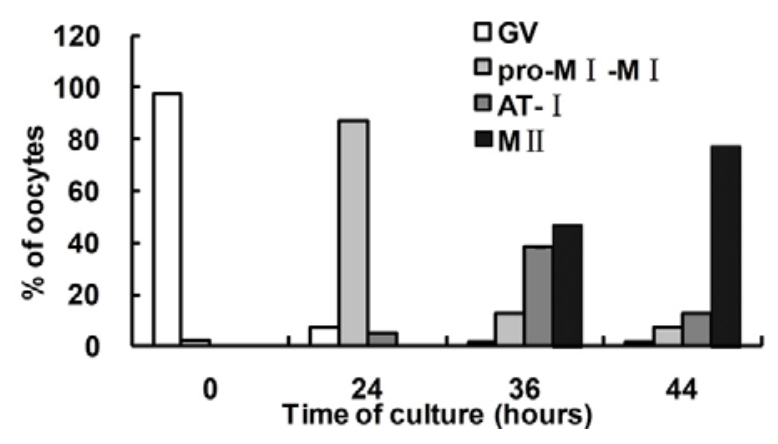

Figure 1. Different meiotic stages of porcine oocytes during in vitro maturation. Each time point was repeated three times. (GV, germinal vesicle; pro-MI, prometaphase I; MI, metaphase I; AT- I, anaphase I and telophase I; MII, metaphase II).

USA). Once $70-80 \%$ of the cells were confluent, $200 \mathrm{ng}$ of the reporter-gene constructs was cotransfected with $30 \mathrm{nM}$ miR-27a mimics or inhibitors using Lipofectamine 2000 (Invitrogen, USA). The scrambles (Ambion, Inc., USA) were transfected as the negative control. After $24 \mathrm{~h}$ of transfection, firefly and Renilla luciferase activities were determined using Promega's dual luciferase assay (Progema, USA). The Renilla luciferase was normalized using the firefly luciferase for correction of transfection efficiency.

\subsection{Statistical analysis}

All of the data used for the statistical analysis were analyzed by the Student $t$-test; $\mathrm{p}<0.05$ was defined as the level of statistical significance.

\section{RESULTS}

\subsection{Meiotic stages of porcine oocytes during in vitro maturation}

Oocytes at different meiotic stages were prepared. As shown in Figure 1, at $0 \mathrm{~h}$ of culture, $97.4 \%$ of the oocytes were at the GV stage. After $24 \mathrm{~h}, 92.6 \%$ of the oocytes underwent GVBD. At the 36-h mark, 38.3\% of the oocytes were at anaphase I or telophase I, whereas $46.7 \%$ were at MII. Finally, by $44 \mathrm{~h}$, the proportion of MII oocytes increased to $77.6 \%$.

\section{2. miRNA expression profiling during porcine oocyte maturation}

To determine whether miRNA biogenesis occurred during porcine oocyte maturation, oocytes at different meiotic stages were prepared according to the results shown in Figure 1. Moreover, the COCs cultured for $36 \mathrm{~h}$ were denuded and cultured for an additional $8 \mathrm{~h}(36+8 \mathrm{~h})$ to evaluate whether cumulus cells affect miRNA expression during late-stage oocyte maturation. Next, these oocytes were examined for miRNA expression using the TLDA system containing 365 mature human miRNAs. As shown in Figure 2A, dynamic changes in miRNA abundance were observed during oocyte maturation. Notably, the total miRNA amount increased by approximately $60 \%$ at $24-36 \mathrm{~h}$ $(\mathrm{p}=0.0007)$, but it was subsequently downregulated to very low levels, only $41 \%$ of the amount at $36 \mathrm{~h}(\mathrm{p}=0.00074$;
Figure 2B). In MII oocytes at $44 \mathrm{~h}, 17$ miRNAs were downregulated, whereas only 1 miRNA was significantly upregulated compared with $\mathrm{GV}$ oocytes at $0 \mathrm{~h}(\mathrm{p}<0.05$; Table 1). However, the oocytes that matured in $36 \mathrm{~h}$ with the cumulus cells and were cultured for an additional $8 \mathrm{~h}$ in the absence of cumulus cells showed miRNA expression profiles very similar to those of oocytes that matured continuously in $44 \mathrm{~h}$ in the presence of cumulus cells. The TLDA results were further confirmed using the TaqMan real-time PCR method (Figure 2C). Moreover, the pathway analysis for the potential target genes of the differentially expressed miRNAs in MII oocytes was conducted and found to be associated with the cellular processes, including the mitogen-activated protein kinase signaling pathway, cell cycle, transforming growth factor- $\beta$ signaling pathway, and Wnt signaling pathway (Figure 2D). Together, these results suggest that miRNA biogenesis occurs during porcine oocyte maturation.

\section{3. miR-27a facilitates the first cell cleavage}

To investigate whether miRNA function was globally suppressed in porcine oocytes, we focused on miR-27a. In breast cancer cells, miR-27a has been reported to facilitate cells undergoing the $\mathrm{G} 2 / \mathrm{M}$ transition and contribute to cell proliferation (14). Thus, if miR-27a activity was not lost in porcine oocytes, it might facilitate the first cleavage. The current study found that miR-27a expression was significantly increased in 2-cell embryos ( $p$ $<0.05$ ) compared with MII oocytes (Figure 3A). Further investigation showed that the first cleavage rates were notably increased $(p<0.01)$ for MII oocytes microinjected with miR-27a mimics but were significantly decreased $(\mathrm{p}<$ 0.05 ) by the miR-27a inhibitor (Figure 3B), indicating that miR-27a facilitated the first cell cleavage.

\section{4. $\mathrm{miR}-27$ a represses $M A P 2 K 4$ translation}

To further test if miR-27a repressed the translation of messenger RNA (mRNA) targets in porcine oocytes, a bioinformatics analysis was used to predict potential target genes of miR-27a. Among the predicted genes, Pig MAP2K4 3'-UTR showed a highly conserved binding site (Figure 4A). Further analysis showed that the endogenous MAP2K4 protein level increased in the 293FT cells transfected with the miR-27a inhibitor but decreased when transfected with the miR-27a mimic, indicating a correlation between miR-27a and MAP2K4 protein expression (Figure 4B). Next, MAP2K4 was demonstrated to be a direct target of miR-27a through cloning of the 3'-UTR into a luciferase reporter vector. The luciferase activity was significantly decreased in the 293FT cells transfected with the miR-27a mimic. In contrast, the miR-27a inhibitor remarkably increased luciferase activity (Figure 4C). To further assess whether miR-27a repressed $M A P 2 K 4$ translation in porcine oocytes, the COCs at the $\mathrm{GV}$ stage were microinjected with an miR-27a mimic or inhibitor, cultured for $44 \mathrm{~h}$, and then analyzed for the presence of the MAP2K4 protein. As shown in Figure 4D, the MAP2K4 protein level significantly increased in the oocytes microinjected with the miR-27a inhibitor but decreased in the oocytes microinjected with the miR-27a 
A
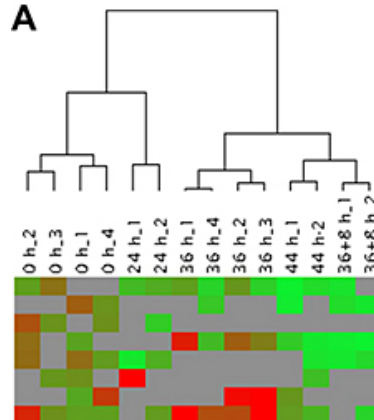

sa.mir.133b-4373172 hsa-miR-194. 4373106
hsa-let-7b-4373168 hsa-miR. $301 \cdot 4373064$ hsa-miR-484-4381032
hsa-miR-148b-4373129 hsa-miR-17-5p-4373119 hsa-miR-191-4373109 hsa-miR-19b-4373098 hsa.mir-20a-4373286 hsa-miR.146b.4373178 hsa.mir.9-4373285
hsa.let.7g-4373163 hsa-miR-186-4373112 hsa-miR-423-4373015 hsa-mir-21-4373090 hsa-mir-320-4373055 hsa-mik-100-4373160 hsa-miR-103-4373158 hsa-miR -24.4373072 hsa.miR.195.4373105 hsa-miR-26a-4373070 hsa-miR-218-4373081 hsa-miR.-92-4373013 hsa-mir-30a-3p-4373062 hsa-mir-125b-4373148 hsa-mik-30c-4373060 hsa-mir-125 a-437314 hs a.miR. 1489.4373130 hsa-miR-30a-5p-437306 hsa-miR-141-4373137 hsa-miR-30e-5p-437305 hsa-mir-30d-4373059 hsa-let-7d-4373166 hsa-miR. 19a-4373099 hsa.mir-374-4373028 hsa-miR-200c-4373096 hsa.mir.210.4373089 hsa-mir-30b-4373290 hsa-mir.93-4373012 hsa-miR-193a-4373107 hsa-miR-27a-4373287 hsa-mir-27b-4373068 hsa-mir-205-4373093 hsa.mir.-204.4373094 hsa-miR-182-4373271 hsa-miR-106b-4373155 hsa-miR-572-4381017 hsa-mir. $187-4373111$ hsa-mir-449b-4381011 sa-mir-335-4373045 hsa-miR-25.4373071 hs $2 \cdot m i R \cdot 338-4373043$ hsa-miR-489.4373214 hsa-miR-660-4380925 hsa-miR-650-438100 hsa-miR.7-4373014 hsa-miR-26b-4373069 hsa-miR-32-4373056 hsa-miR-646-4381002 hsa.mir. $30 \mathrm{e}-3 \mathrm{p}-43730 \mathrm{~s}$
B

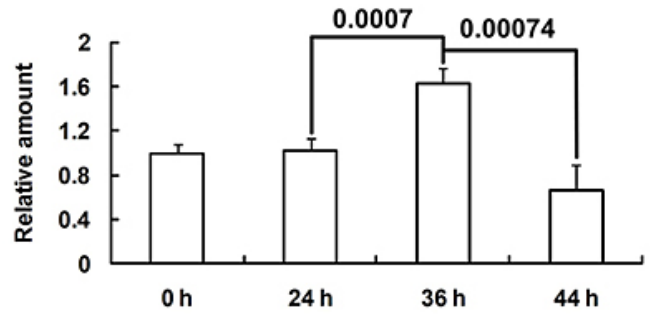

C

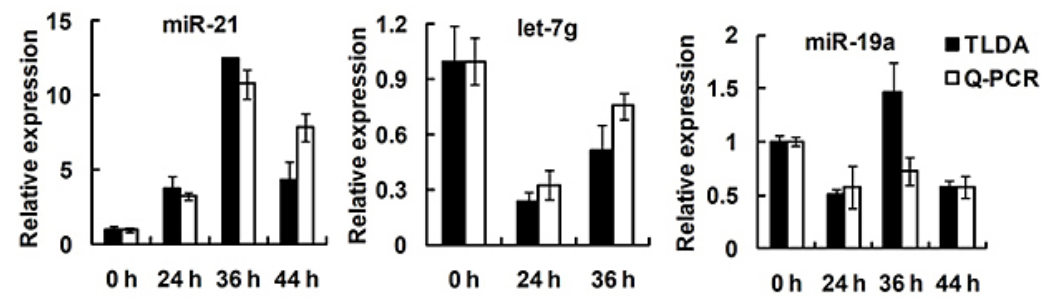

D

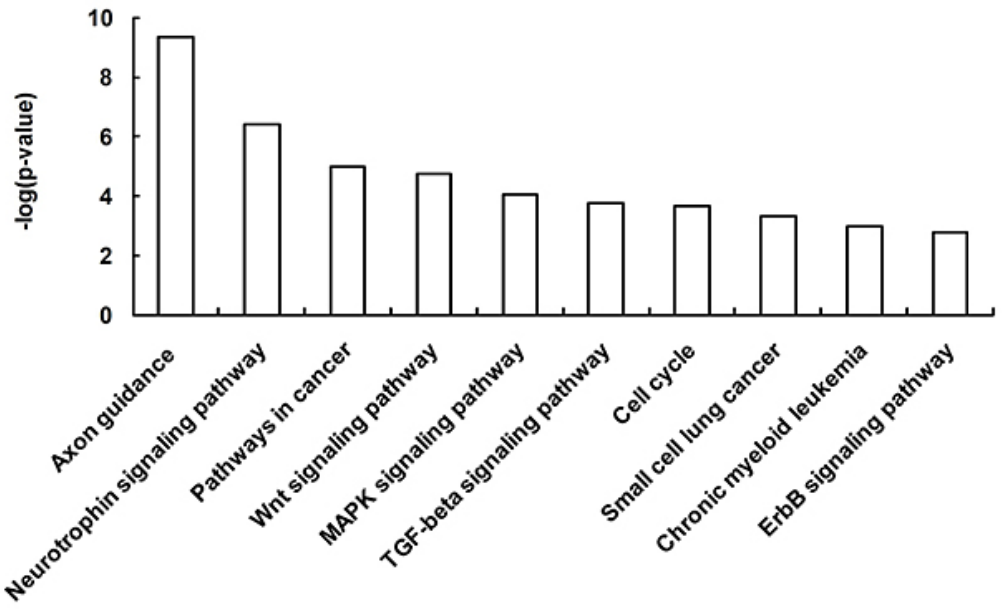

Figure 2. miRNA expression profiles during porcine oocytes maturation. (A) Hierarchical clustering of relative miRNA expression of porcine oocytes at $0,24,36$, and 44 hours of culture. Rows represent the relative expression levels for a single miRNA; columns show the expression levels for a single sample. The red or green color indicates relative high or low expression, respectively, while gray indicates absent data points. (B) Total relative amount of miRNAs during porcine oocytes in vitro maturation. It was calculated based on the relative miRNAs levels obtained from TLDA. Column, mean; bar, S.D. (C) TLDA results were validated using Taqman real-time PCR. Relative levels of let-7g, miR-21, and miR-19a during porcine oocyte maturation were analyzed using real-time PCR or TLDA. Column, mean; bar, S.D. (D) The top 10 signaling pathways for putative target genes of miRNAs differently expressed when $44 \mathrm{~h}$ VS. $0 \mathrm{~h} . \mathrm{p}<0.05$ was defined as statistically significant.

mimic, suggesting that miR-27a was able to repress target translation.

\section{DISCUSSION}

To our knowledge, this is the first study to examine miRNA biogenesis and activity during porcine oocyte maturation. We found that miRNA expression underwent dynamic changes during oocyte maturation. In addition, 18 miRNAs were found to be differentially expressed in MII oocytes (Table 1), and the predicted targets of these miRNAs were mostly related to development (Figure 2D). Moreover, by examining the effect of miR-27a on the first cleavage and its ability to repress $M A P 2 K 4$ translation, we found that miR-27a activity was not suppressed in porcine oocytes.

This finding 


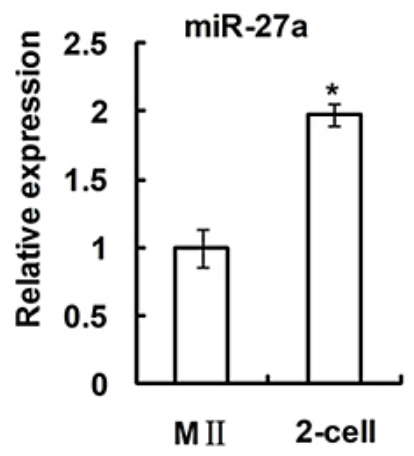

A
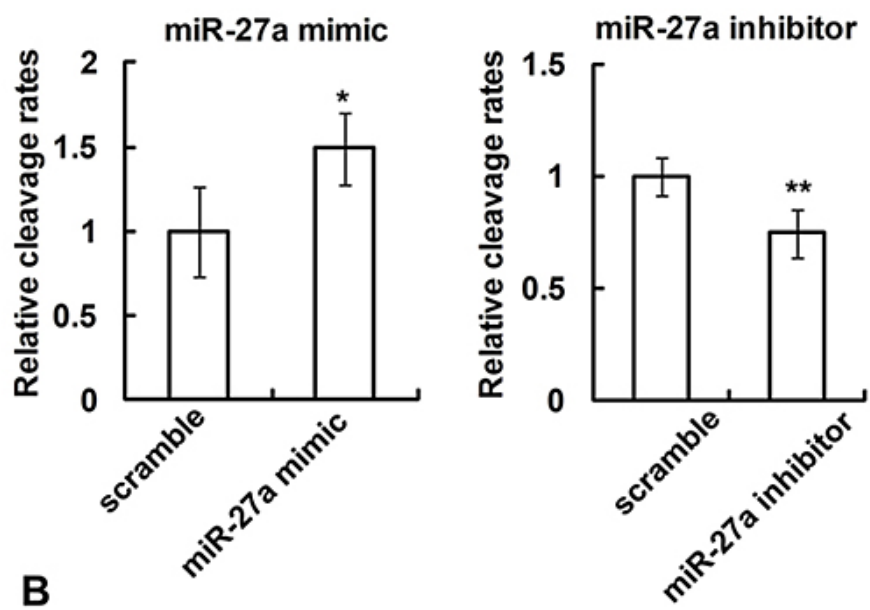

Figure 3. miR-27a facilitates the first cell cleavage. (A) Relative expression of miR-27a in MII oocytes and two-cell stage embryos were determined by real-time PCR. Column, mean; bar, S.D. (B) Cleavage rates were analyzed for oocytes microinjected with the miR-27a mimic or inhibitor. Scramble was used as the negative control miRNA. Each group was repeated for three times. Column, mean; bar, S.D.

A

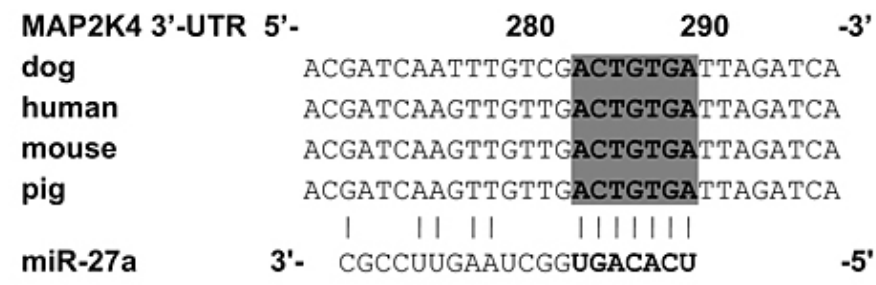

C
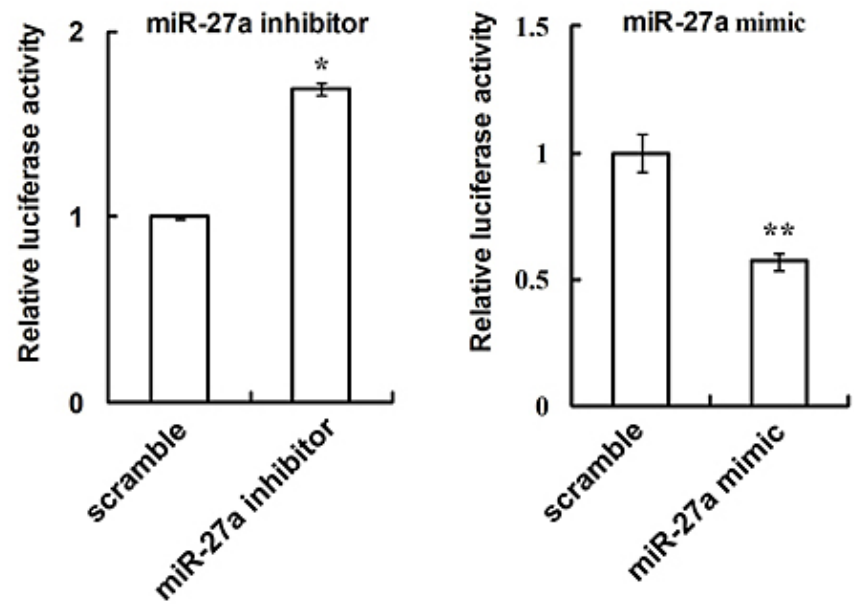

B
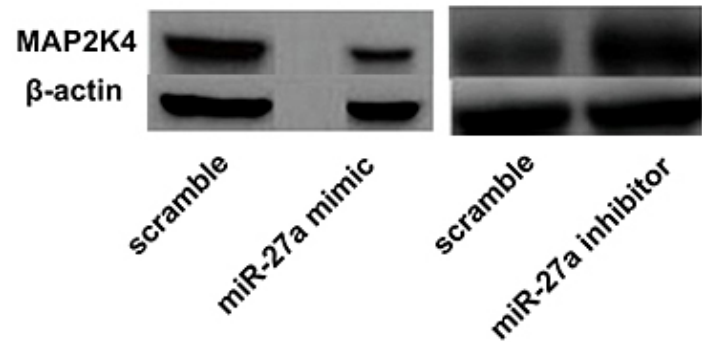

D
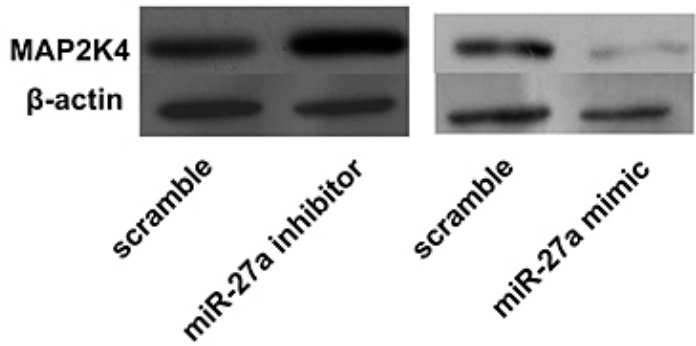

Figure 4. miR-27a represses translation of MAP2K4. (A) Conserved miR-27a binding site within the MAP2K4 3'-UTR of mammals. (B) Expression of MAP2K4 was determined by Western blot after cells were transfected with the miR-27a mimic or inhibitor. Scramble was used as the negative control miRNA. $\beta$-actin was shown as a loading control. (C) Relative luciferase activities in 293FT cells. Reporter vector containing pig MAP2K4 3'-UTR was cotransfected with the miR-27a mimic or inhibitor. Scramble was used as the negative control miRNA. Columns, mean; bar, S.D. ${ }^{*}, \mathrm{p}<0.05 ;{ }^{* *}, \mathrm{p}<0.01$. (D) Expression of MAP2K4 was determined by Western blot after oocytes were microinjected with the miR-27a mimic or inhibitor. Scramble was used as the negative control miRNA. $\beta$-actin was shown as a loading control. 
Table 1. List of differentially expressed miRNAs in MII oocytes compared with GV oocytes

\begin{tabular}{|l|l|l|l|l|}
\hline Number & miRNA detector & Fold changes (MII/GV) & p-value (p<0.05) \\
\hline Up-regulated miRNAs & miR-16 & 0.67 & 0.00219 \\
\hline 1 & miR-31 & 0.46 & 0.0031 \\
\hline 2 & miR-30c & 0.60 & 0.00348 \\
\hline 3 & miR-92 & 0.44 & 0.00399 \\
\hline 4 & miR-148a & 0.58 & 0.00615 \\
\hline 5 & miR-210 & 0.33 & 0.00969 \\
\hline 6 & miR-301 & 0.29 & 0.01399 \\
\hline 7 & miR-133b & 0.20 & 0.01617 \\
\hline 8 & miR-106b & 0.76 & 0.01911 \\
\hline 9 & miR-484 & 0.20 & 0.01946 \\
\hline 10 & miR-140 & 0.65 & 0.01952 \\
\hline 11 & miR-26a & 0.39 & 0.02031 \\
\hline 12 & let-7d & 0.53 & 0.02451 \\
\hline 13 & miR-30a-5p & 0.64 & 0.03036 \\
\hline 14 & miR-335 & 0.67 & 0.03561 \\
\hline 15 & miR-24 & 0.64 & 0.04154 \\
\hline 16 & miR-93 & 0.51 & 0.04206 \\
\hline 17 & miR-21 & 4.32 & 0.03377 \\
\hline Down-regulated & & \\
\hline 1 & & & \\
\hline
\end{tabular}

is in contrast to those of recent studies on mouse oocytes and early embryos in which miRNA activity is globally suppressed $(10,11)$. This difference might be due to species differences. Thus, miRNA mechanisms underlying porcine oocyte development might differ from those of mouse oocytes. However, in the current study, we examined only the miR-27a activity; thus, we cannot exclude the possibility that miRNAs other than miR-27a lose their activities in porcine oocytes. Based on this aspect, further investigations are definitely required. However, it is a fact that miRNA biogenesis and changes occur during porcine oocyte maturation.

Oocyte maturation is accompanied by mRNA deadenylation and degradation. In mouse oocytes, approximately $85 \mathrm{pg}$ of mRNA exists at the GV stage, but $30 \%$ of this amount undergoes degradation during oocyte maturation (15) and the protein synthesis rate also decreases after GVBD. These mRNAs are thought to be post-transcriptionally regulated, typically through motifs embedded in the $3^{\prime}$-UTR (16). Furthermore, miRNA is a post-transcription regulator that results in mRNA degradation or translation inhibition by binding the target site embedded in the 3'-UTR. It might be universal for miRNA to mediate clearance of maternal mRNA in the regulation of maternal to zygotic transition (17). For example, in zebrafish, miR-430 was observed to accelerate the deadenylation and clearance of maternal mRNA and was therefore critical for the maternal-to-zygotic transition (17). This mechanism might be suitable for oocyte maturation as well. Thus, in this study, the total amount of miRNA that increased in the oocytes at $36 \mathrm{~h}$ might be related with the deadenylation and clearance of mRNA accumulated during oocyte growth, thus resulting in the decreased rate of protein synthesis after GVBD. After mRNA is deadenylated and degraded, miRNAs might not be required; thus, in our study, the miRNA level was lowest at $44 \mathrm{~h}$. Therefore, dynamic miRNA changes during porcine oocyte maturation might be related with deadenylation and degradation of maternal mRNA, thus enhancing the maturation process.

Cumulus cells are a group of granulose cells that surround the oocyte in the antral follicle and presumably participate in meiotic arrest, meiotic resumption, and cytoplasmic maturation (18). Removal of the cumulus cells before in vitro maturation was deleterious to oocyte maturation and decreased the sperm penetration rates in mice, rats, and cattle $(19,20)$. However, in pigs, after the COCs were cultured for $36 \mathrm{~h}$, the miRNA expression profiles were similar between oocytes cultured with $(44 \mathrm{~h})$ and those cultured without cumulus cells $(36+8 \mathrm{~h}$; Figure 2A). It is likely that the junctions between the cumulus cells and the oocytes were disconnected after $36 \mathrm{~h}$ of in vitro maturation; thus, cumulus cells may not be required for late-stage oocyte maturation as far as miRNA expression is concerned. It has been reported that cumulus-oocyte communication changed dynamically during porcine oocyte maturation in vitro. Between the MI $(0-24 \mathrm{~h})$ and MII $(36-44 \mathrm{~h})$ stages, cumulus-oocyte transzonal projections became disconnected because of cumulus expansion (21). Thus, the similar expression profile in our study might also be the result of cumulus expansion. To our knowledge, this is the first study to examine the relationship between oocytes and cumulus cells from the viewpoint of miRNA expression.

In conclusion, by observing the miRNA biogenesis and the miR-27a function in the first cleavage and the repression of $M A P 2 K 4$ translation, we speculate that miRNA activity might not be globally suppressed in porcine oocytes. However, further experiments are definitely required to confirm this point.

\section{ACKNOWLEDGMENTS}

This work is supported by the National Basic Research Program of China (Project Number: 2007CB947402) and National High Tech Research Program of China (Project Number: 2010AA10A103).

\section{REFERENCES}

1. Kume, S., T. Endo, Y. Nishimura, K. Kano \& K. Naito: Porcine SPDYA2 (RINGO A2) stimulates CDC2 activity and accelerates meiotic maturation of porcine oocytes. Biology of reproduction, 76, 440 (2007)

2. Ayalon, D., A. Tsafriri, H. R. Lindner, T. Cordova \& A. 
Harell: Serum gonadotrophin levels in pro-oestrous rats in relation to the resumption of meiosis by the oocytes. Reproduction, 31, 51 (1972)

3. Sun, Q. Y. \& T. Nagai: Molecular mechanisms underlying pig oocyte maturation and fertilization. Journal of Reproduction and Development, 49, 347-359 (2003)

4. Liang, C. G., Y. Q. Su, H. Y. Fan, H. Schatten \& Q. Y. Sun: Mechanisms regulating oocyte meiotic resumption: roles of mitogen-activated protein kinase. Molecular Endocrinology, 21, 2037 (2007)

5. Bartel, D. P.: MicroRNAs: Genomics, Biogenesis, Mechanism, and Function. Cell, 116, 281-297 (2004)

6. Gaur, A., D. A. Jewell, Y. Liang, D. Ridzon, J. H. Moore, C. Chen, V. R. Ambros \& M. A. Israel: Characterization of microRNA expression levels and their biological correlates in human cancer cell lines. Cancer research, 67, 2456 (2007)

7. Tang, F., M. Kaneda, D. O'Carroll, P. Hajkova, S. C. Barton, Y. A. Sun, C. Lee, A. Tarakhovsky, K. Lao \& M. A. Surani: Maternal microRNAs are essential for mouse zygotic development. Genes \& development, 21, 644 (2007)

8. Tesfaye, D., D. Worku, F. Rings, C. Phatsara, E. Tholen, K. Schellander \& M. Hoelker: Identification and expression profiling of microRNAs during bovine oocyte maturation using heterologous approach. Molecular reproduction and development, 76, 665-677 (2009)

9. Nakahara, K., K. Kim, C. Sciulli, S. R. Dowd, J. S. Minden \& R. W. Carthew: Targets of microRNA regulation in the Drosophila oocyte proteome. Proceedings of the National Academy of Sciences of the United States of America, 102, 12023 (2005)

10. Ma, J., M. Flemr, P. Stein, P. Berninger, R. Malik, M. Zavolan, P. Svoboda \& R. M. Schultz: MicroRNA activity is suppressed in mouse oocytes. Current Biology, 20, 265-270

11. Suh, N., L. Baehner, F. Moltzahn, C. Melton, A. Shenoy, J. Chen \& R. Blelloch: MicroRNA function is globally suppressed in mouse oocytes and early embryos. Current Biology, 20, 271-277

12. Sun, Q. Y., L. Lai, A. Bonk, R. S. Prather \& H. Schatten: Cytoplasmic changes in relation to nuclear maturation and early embryo developmental potential of porcine oocytes: Effects of gonadotropins, cumulus cells, follicular size, and protein synthesis inhibition*. Molecular reproduction and development, 59, 192-198 (2001)

13. Wei, H., Q. Li, J. Li, Y. Li, Y. Dai, Y. Ma, K. Xue \& N. Li: Effect of leptin on oocyte maturation and subsequent pregnancy rate of cloned embryos reconstructed by somatic cell nuclear transfer in pigs. Progress in Natural Science, 18, 1583-1587 (2008)

14. Mertens-Talcott, S. U., S. Chintharlapalli, X. Li \& S. Safe: The oncogenic microRNA-27a targets genes that regulate specificity protein transcription factors and the G2-M checkpoint in MDA-MB-231 breast cancer cells. Cancer research, 67, 11001 (2007)

15. Paynton, B. V., R. Rempel \& R. Bachvarova: Changes in state of adenylation and time course of degradation of maternal mRNAs during oocyte maturation and early embryonic development in the mouse* 1 . Developmental biology, 129, 304-314 (1988)

16. Evsikov, A. V., J. H. Graber, J. M. Brockman, A. Hampl, A. E. Holbrook, P. Singh, J. J. Eppig, D. Solter \& B. B. Knowles: Cracking the egg: molecular dynamics and evolutionary aspects of the transition from the fully grown oocyte to embryo. Genes \& development, 20, 2713 (2006)

17. Giraldez, A. J., Y. Mishima, J. Rihel, R. J. Grocock, S. Van Dongen, K. Inoue, A. J. Enright \& A. F. Schier: Zebrafish MiR-430 promotes deadenylation and clearance of maternal mRNAs. Science, 312, 75 (2006)

18. Tanghe, S., A. Van Soom, H. Nauwynck, M. Coryn \& A. De Kruif: Minireview: Functions of the cumulus oophorus during oocyte maturation, ovulation, and fertilization. Molecular reproduction and development, 61, 414-424 (2002)

19. Vanderhyden, B. C. \& D. T. Armstrong: Role of cumulus cells and serum on the in vitro maturation, fertilization, and subsequent development of rat oocytes. Biology of reproduction, 40, 720 (1989)

20. Fukui, Y. \& Y. Sakuma: Maturation of bovine oocytes cultured in vitro: relation to ovarian activity, follicular size and the presence or absence of cumulus cells. Biology of Reproduction, 22, 669 (1980)

21. Suzuki, H., B. S. Jeong \& X. Yang: Dynamic changes of cumulus-oocyte cell communication during in vitro maturation of porcine oocytes. Biology of reproduction, 63, $723(2000)$

Key Words: MicorRNA, Porcine Oocyte, Meiotic maturation, $M A P 2 K 4$, microRNA-27a

Send correspondence to: Ning Li, State Key Laboratory for Agrobiotechnology, China Agricultural University, Beijing 100193, China, Tel: 86-10-62733342, Fax: 86-10-62733342, E-mail: kuaileqianjin@gmail.com 